Creusot, qui pourra envoyer ses produils métallurgiques en llalıe par une route qui ne sera plus celle de l'école buissonnière; ce seront loutes les régions commerçantes, industrielles et agricoles du bassin de la Loire, de la Saône et de la Côte-d'or, qui pourront diriger directement leurs produıts sur Ja Lombardse et le Plémont. D'autre part, les importantes ressources minières de la vallée d'Aosle trouveraient un écoulement facile vers les bassins houillers français, bassıns qui manquent tolalement en Italie.

Le projet Sléphanı-Pıcquet offre de grandes facilıtés lechniques. Le percement du Monl-Blanc serait effeclué en effet à la hauteur (le 1.050 mètres, sur une longueur de $13,5 \mathrm{kms}$, à travers une couche puremenl granitıque, et les obstacles seraient réduits au minımum.

Ce tracé donne les plus faibles déchivités, les pentes ne dépassent pas $15 \mathrm{~mm}$ et les courbes n'ont pas moins de 500 mètres de rayon. Le développement de la ligne Aoste-Chamonix est de $52 \mathrm{kms}$. De Chamonıx à l'entrée du grand tunnel, le tracé parcourt $6,2 \mathrm{kms}$, dont $3 \mathrm{~km}$. avec la pente de $0 \mathrm{~m}$. 0016 par mètre. Il pénètre dans le grand lunnel au km. 6,2, à l'altilude de 1.050 mètres, et franchit le point culminant au $\mathrm{km}$. 13,8 à 1.080 mètres d'altitude. Il descend ensuile avec une pente de 4,8 pour 1.000 , pour sortir un peu en amont de Courmayeur, au km. 19,7, à 1.050 mètres d'altitude, et atteint la station de Courmayeur au $\mathrm{km}$. 20,4. Il franchit la Doire sur un viaduc de 205 mètres de longueur, et après avoir traversé 3 tunnels mesurant ensemble 1.700 mètres, il atteint la station de Morgex au km. 26,5, à l'altitude de 954 mètres. Il traverse ensuite 3 tunnels, d'une longueur lotale de 1.450 mètres, franchit la Doire sur un pont de 125 mètres de longueur, tràverse encore un tunnel de 346 mètres et franchit de nouveau la Doire sur un viaduc de 130 mètres de longueur; il parcourt ensuite 6.500 mètres avec une pente de 1,48 pour 100 , et atteint la station de Villeneuve au km. 42, à 740 mètros d'əllitude. Après avoir traversé 3 lunnels d'une longueur totale de 1.709 mètres, il franchıt encore la Dorre sur un viaduc de 200 mètres, puis parcourl ensuite $11 \mathrm{kms}$ avec la pente de 1,27 pour 100 , et atteint enfin la statıon terminus d'Aoste, au km. 52, à 600 mètres d'allitude, après avoir traversé trois tunnels mesurant ensemble 1.755 mètres.

Le souterrain de faîte serait presque entièrement placé dans le granil talqueux (protagıne de Saussure), et c'est à peine s'il existera 1500 mètres de chaque côté en terrain métamorphique, sauf, bıen entendu, l'existence possible, mais peu probable, d'ırruptıons intérieures qui seraient serpentınes.

C'est là un avantage indiscutable, et d'une grande valeur sur toutes les autres percées qui ont à pénétrer, ou qui ont pénétré des roches métamorphiques amphiholiques, ou serpentineuses, d'une extrême dureté.

La protagine est une roche pleine, el, à en juger d'après lusage que l'on en fait, c'est un granit relativement faclle à travalller, ce qui est un avantage par rapport au Gothard et au MontCenis.

Le souterrain du Gothard a été construit à $2.800 \mathrm{fr}$. le mètre, non compris la vole et les maçonneries, solt 3.100 à 3.200 francs avec celte additıon, et le chemin complet est revenu à 3.800 francs le mètre.

Mais en tenant compte des progrès réalisés par l'ınvention des perforatrices hydrauliques et électriques, et en se basant sur la construction du Simplon, on peut, aujourd'hui, évaluer à 2700.000 francs par kilomètre la construction des souterrains dans le genre de ceux du Mont-Blanc, à 500.000 francs par kilomètre pour les aulres passages à cıel ouvert et les ouvrages d'art.

La dépense totale, d'après les calculs faits par les auleurs du projet, ne dápasserait pas 93 millıons de francs, et celte somme est paraît-il déjà garantıe par une banque française.

Voici d'alleurs comment se répartil l'estimation des dépenses :

Percement el installation du tunnel, $13 \mathrm{kms} 5$ à

$2.700 .000 \mathrm{fr}$. par km................... $3644000 \mathrm{fr}$.

Construetion de $38 \mathrm{kms}$ de voie d'accès, y compris les ouvrages d'art, à raison de $500.000 \mathrm{fr}$. par

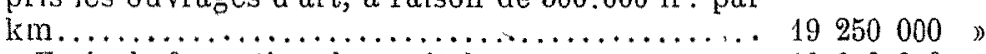

Frais de formation du capital.............. 10000000

Intérêt du capital à 5 pour 100 , pendant le temps

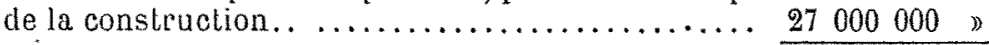

Tutal........................

Les travaux de percement exigeraient une pérıde de lemps de cinq ans, et le tunnel pourrait être livré à l'explortation dans un délai de cinq ans et demi, à partır du jour de la mise à exécution des travaux.
Les chules de l'Arve et de la Doire fourniraient, comme au Simplon, l'énergie requuse pour le percement des tunnels, puis ensuite le courant nécessaire à la traction électrique des trains.

H. Bellet.

\section{LA SCIENCE ET L'INDUSTRIE}

Discours prononcé au Congrès de Lyon de l'Association Française pour l'Avancement des Sciences, par M. LippmanN, membre de l'Institut, président de l'Association.

Le jeudi, 2 août r 1906, s'est ouvert, à Lyon, dans le grand Amphithéâtre de la Faculte des Sciences, le trente-cinquième congrès de l'Association Françatse pour l'Avancement des Sciences. Après le discours de réception du maire de Lyon, M. Lrppmann, membre de l'Institut, Président de l'Association, prononce le discours d'usage sur le rôle de la science caans l'industrie. Après avorr remercié les Autorités et le Comité local d'organisation, et après avoir souhaité la bienvenue aux savants étrangers, M. Lippman aborde la partie principale de son discours.

Lyon possède toutes les attractions d'un grand centre intellectuel. En premier lieu, une belle université qui a la pleıne conscience et la claire intelligence de sa tâche. La science et l'industrie vivent ici en harmonı. On trouve dans cette région des usines scientifiquement organisées, où l'on a compris que la science doit apporter au développement industriel un secours journalier. C'est là une vérité méconnue dans d'autres parties de la France. On rencontre trop souvent cette erreur que l'industrie n'a besoin que de techniciens, ou du moins qu'elle peut se contenter de sciences dites appliquées, enseignées spécialement en vue des diverses industries. Ce serait la science réduite à sa partie vraiment utile - ceci, on n'ose pas le dire, mais on le pense - et Pasteur n'a pas manqué de souligner l'ineptie dangereuse qui se cache parfois sous ce terme de science appliquée. Vollà l'erreur néfaste qui al fait tomber l'industrie françaıse, comme production globale, du premier au quatrième rang. C'est un devoir de faire la guerre aux idées fausses quand on les rencontre, et de leur substituer la vérité salutarre. Pour cette raison, je vous demande la permission de vous présenter aujourd'hu1 quelques observations sur cette question des relations entre la science et l'industrie. Si je n'apprends guère à mes auditeurs que ce qu'ils savaient déjà, je les prie de m'excuser. Je désire simplement faire appel à l'influence de l'Association Française pour répandre davantage certaines vérités qui touchent à la science et dont l'oubl 1 coûte terriblement chèr à notre pays. C'est le rôle même que notre Association est destinée à jouer.

11 est facile de définır le lien qui unit la science à $l$ industrie.

Il n'y a qu'une nature. Les forces qui constituent le monde sensible sont celles mêmes qui animent les apparells de nos laboratoires et que l'on utilise dans l'industrie, dans les arts de la parx er de la guerre. Il n'y a donc qu'une science, laquelle n'est ni professorale, ni industrielle, ni civile, ni militaire.

La science expérimentale est l'art de manier méthodiquement les forces de la nature. Aussi l'industrie et la science se sont-elles développées parallèlement.

Pendant un nombre inconnu de siècles, la science a été empirique et l'industrie réduite à des tâtonnements. Depuis environ cent ans, la science s'est développée plus que pendant les milliers d'années qui précédaient et l'industrie a marché à pas de géant. Les anciennes industries sont totalement transformées et d'autres sont nées, auxquelles on ne pensait pas autretois. Les industries chimiques et électriques par exemple. Jadis la téléphonie n'eût pu figurer que dans un conte de fée. $\mathrm{Si}$ les industries d'autrefois paraissent faibles et barbares auprès de celles de nos jours, il serait injuste cependant d'en parler avec dédain. Quelle sagacité d'observation et quelle patience géniale il a fallu pour les créer sans le secours de la théorie! 
On connait d'ailleurs assez mal leur hıstoire. On ne sait même pas quel fut le mécanicien merveilleux qui a créé la première roue. Nous 1gnorons tout de cet ancêtre qui, un beau jour, s'est mis à faire du feu Il n'est pas sûr qu'il s'appelât Prométhée. Et après tout, qu'importe? Son œuvre reste, et luimême ne nous serait pas moins inconnu si nous savions pro. noncer les syllabes de son nom.

Il est évident d'autre part que le travail industriel est assujettı à d'autres conditions que les recherches de laboratoire. Le prix de revient importe, et je n'insiste pas, je veux dire qu'il faut pour qu'un procédé devienne industriel, que la mise en œuvre en ait eté étudiée dans tous ses détails, depurs la construction des appareils et le choix des matériaux, jusqu'au tour de main de l'ouvrier ; rien n'est indifférent. Chauffe-t-on à la houille, 1l faut chorsir la qualité du combustible; et le coup de pelle du chauffeur habile a une valeur qui se chiffre Dans cette formation détaillée et pratique, le tâtonnement, l'empirisme reprennent leur importance

Des années sont nécessaires parfois pour constituer une industrie compliquée, des années sont nécessaires pour la connaître à fond; quand on voit de près certaines industries on est surpris de la somme d'expérience variée et d'attention assidue qu'il faut à un homme intelligent pour apprendre complètement le métier.

Un personnel technique bien instruit est donc indispensable à l'usine; il pourra' avoir été préparé dans les écoles spécrales, mais il devra apprendre son métrer sur place, à l'atelier, car on ne l'apprend pas ailleurs.

L'organisation de l'usine sera technique également, et pourvue par exemple de bureaux d'essais qui fonctionneront avec une régularité automatique.

Le directeur est tranquille une fors que la marche régulière de l'exploitation est assurée par l'étude de tous les détalls. Sans doute; mais dans l'industrie, on n'est jama1s tranquille. Quand tout a été prévu, il reste l'imprévu, aujourd'huı est assuré; mais demain viendra, et demain c'est l'iconnu. Il y a l'accident heureux ou malheureux qu'll faut fixer ou prévenir. Il y a surtout, et cela d'une manière permanente, la concurrence, celle des producteurs du même pays et celle de l'industrie étrangère. L'industrie est une lutte sans fin et sans trêve, où la guerre civile est mêlée à la guerre étrangère. On attaque hardiment, on démolit assidûment ses rivaux, et si so1-même l'on ne veut pas succomber, l'on est tenu de perfectionner en temps utile sa tactique et son armement.

C'est de la même façon qu'un pays a beau posséder une artillerie dont il a le droit d'être fier, au bout de quelques années, 11 est forcé de renvoyer à la fonderie des batteries presque neuves, afin de remplacer cette belle artillerie par une autre plus belle encore, à cause de la concurrence.

Mais, du moment que des éléments nouveaux s'introduisent dans la fabrication, les procédés acquis sont remis en question, et l'expérience technique devient insuffisante.

Supposons que des métaux tels que le molybdène et le titane viennent s'allier à l'acier, le bureau des essais ne dit plus rien, car un essai n'est pas une analyse; force est d'avoir recours à un laboratoire d'analyse complet et à un chimiste complet qui ne craigne pas les métaux rares. A la chimie devra s'adjoindre la physico-chımie munie de ses méthodes les plus subtiles. Dès l'instant que l'on est amené à sortir de la routine, on tombe dans le domaine de l'imprévu, de l'inattendu, réservé aux chercheurs et aux savants.

Toutes les ressources de l'analyse mathématique et de l'expérimentation peuvent être mises en réquisition. On ne peut combiner un objectıf nouveau sans l'arde des mathématiques. On sait que la télégraphie transatlantique fut sauvée par lord Kelvin qui trouva par l'analyse la cause et le remède de la singulière lenteur avec laquelle les signaux électriques traversent un câble immergé. Le même physicien montra comment on doit calculer le circuit de distribution d'un courant alternatif. De nouveaux instruments ont fait successivement leur entrée dans l'industrie. Le microscope fut introduit par Pas- teur dans l'œnologie el dans l'élevage des vers à soie; le spectroscope servit pour le procédé Bessmer. On tire un obus de marme contre une currasse d'acier, 1 l s'y loge en un millième de seconde, et le fabricant veut savorr par quelles phases a passé le refoulement de l'acier pendant que l'obus a falt son geste brutal On emprunte alors à l'acoustıque une méthode délicate : celle par laquelle Mach a photographié instantanément la compression de l'air autour des branches d'un diapason.

On pourrait citer nombre d'exemples analogues empruntés à la physique. En chimie, le fait est encore plus évident, la fabrication se transforme fréquemment. les corps nouveaux se multiplient et une partie des 5000 substances que découvre annuellement la chimie organique est brevetée et mise dans le commerce.

Conclusion - tant qu'une fabrication se résigne à rester statıonnaire, elle peut se contenter d'un personnel technique expérimenté et d'une organisation automatique La nécessité des progrès se fait-elle sentir? Il faut un personnel scientifique pourvu de laboratoires de recherches installés à l'usine.

Telle est, en effet, la méthode suivie par d'autres nations qui ont progressé plus rapidement que nous. C'est l'Allemagne qui a eu le mérite de donner l'exemple. Nos vorsins d'outre-Rhin appliquent largement le système que je viens d'indiquer et 1 ls en sont largement récompensés. C'est ansi que la ma1son Zeiss d'Iéna, a I4 docteurs ès-sciences a son scrvice, tant mathématiciens que physiciens Les grandes fabriques de couleurs d'aniline et de produits organiques de même pays emplorent plus de chimistes scentifiques que de techniciens : ainsi l'une d'elles utilise 55 chimistes scientifiques pour 31 techniciens; une autre 145 chimistes scientifiques pour 175 techniciens, une troisième 148 chimistes scientifiques pour 75 techniciens. Les laboratoires de recherches sont dans l'usine et grandement installés; l'un d'eux possède une bibliothèque de 14.000 volumes, un autre use pour 125.000 francs par an de verrerie chimique Tout cela côte cher sans doute, mais ces grandes fabriques donnent de 20 à $33 \%$ de dividendes aux actionnaires.

Toute substance nouvellement découverte et utilisable est aussitôt brevetée, ainsi que ses succédanés que l'on recherche systématiquement; les brevets se comptent par milliers. La maison Baeyer en possède 1000 dans son pays el 1200 à l'étranger. Il s'établit ainsi un monopole de fait. L'Allemagne exporte en 1904 pour 156.000 .000 de couleur d'aniline, c'est-àdire 195 fors plus que la France. Sa méthode est donc bonne et la nôtre mauvaise. Les millions qu'elle dépense généreusement pour ses mathématiciens, ses physiciens, ses chimistes, pour ses laboratoires de recherches installés à l'usine, lui valent un bénéfice brut de 1.250 .000 .000 annuellement. C'est un placement intelligent. Et en outre, l'Allemagne y gagne d'exercer une influence mondiale par ses universités, qui fournisscnt aux usines d'Europe et d'Amérique une grande partie du personnel savant dont elles ont besoin.

Les Américains savent employer les savants. L'année dernière je recevais la visite d'un homme de science qu'une très grande Compagnie de construction à New-York a mis à la tête de ses services; il faisait le tour des universités d'Europe avant de se construire, aux frais de sa Compagnie, un laboratoire de recherches qu'il cntendait installer supérieurement. Il me donna sur la distinction qu'il fait également entre le. personnel savant et le personnel technique, un détail piquant. Il paraît que les techniciens américains ont la manie d'essayer sans permission des variantes, en vue souvent de trouver un perfectionnement brevetable ;ils sortent ainsi de leur rôle. Aussi remplace-t-on quand faire se peut, les techniciens par des techniciennes. Il paraît que l'on trouve là-bas en nombre suffisant des femmes instruites et capables; de plus ces Américaines sont dociles; elles ne se permettent jamais d'inventer quelque chose à l'usine.

D'autres pays, l'Autriche, la Suisse par exemple, ont encore adopté la même méthode. Ce sont des pays pourvus d'univer- 
sités autonomes, et ils leur empruntent leur personnel scientifique. A cette liste, ajoutons la Russie. Ce pays possède d'immenses ressources et les Russes appliquent déjà la bonne méthode pour les exploiter; ils n'ont point là-dessus d'idées fausses Aussi a1-je entendu dire par des personnes compétentes qu'une fois le calme rétabli, au bout d'un nombre limité d'années, la Russie viendra prendre rang après l'Allemagne, c'est-à-dire avant nous.

Chez nous les Idées sont toutes différentes On se préoccupe rarement de pourvoir une usine du personnel savant. On n'y installe pas de mathématiciens, peu de physiciens, trop peu de chimistes. Les propriétaires d'usines ne veulent pas entreprendre cette dépense, ou bien, leurs actionnaires ne sont pas disposés à leur accorder lics crédits nécessaires. Ils admettent le technicien, mais renvoient le chercheur au laboratorre de la faculté. L'esprit public est routinier et sceptique en matière d'innovations scientifiques. Il n'y a qu'à rappeler comment ll a accueilli les grandes inventions, celles de la, vapeur par exemple. Papin pourtant était français, ainsi que Sadi-Carnot qui découvrit la théorie générale de la machine à vapeur et des moteurs thermiques, et fonda cette thermodynamique dont relèvent toutes les sciences expérimentales, tant physiques que biologiques. Cependant le grand public est resté pendant près d'un demi-siècle aveugle et sourd devant ce fait évident, que la vapeur était de première importance pour l'industrie; il fut impossible de lu apprendre que les moteurs à vapeur anımaient les fabrıques anglaıses, et commençaient l'immense fortune de l'Angleterre. Carnot soulignait en 1832 l'emploi de la vapeur en Angleterre; on ne l'écouta pas.

La télégraphie électrique est d'origine française puisqu'elle remonte à Ampère. Nous constatons que le public conserva à son égard la même ignorance. Et la série continue. Il me souvient qu'un jour en I870 - j'étais à l'Ecole Normale, mon maître M." Sainte-Claire Deville eut la bonté de m'appeler avec mes camarades à son laboratoire, pour nous montrer un petit appareil qu'il avait installé; je ne connaissais cet instrument que par sa description et $\mathrm{j}$ 'avais peine à $\mathrm{y}$ croire; mais je le portai à mon oreille, j'entendis, et je fus émus, c'était le téléphone. Dix ans plus tard, en I88I, l'exposition d'électricité s'ouvrait aux Champs-Elysées, le public y afflua; on faisait queue pour entendre le téléphone, le théâtrophone, installés dans de grandes salles par des Compagnies américaines. De fait, le téléphone, fut découvert par le public parisien en $188 \mathrm{r}$. Ce petit instrument avait mis dix ans à franchir la grille de fer qui sépare la rue d'Ulm du laboratoire de Deville. La machine Gramme, la lampe Edison, le phonographe eurent la même fortune que lui et émerveillèrent les visiteurs de l'exposition de I88I.

Jamais peuplade asiatique n'accueillit avec une plus franche admiration les inventions apportées de loin par des barbares d'Occident.

Telle a été, telle est encorc la mentalité du grand public, de cette classe moyenne qui a passé par le lycée. Cette masse dirigeante a ses qualités, elle est très civilisée, honnête, passablement lettrée et plus artiste peut-être qu'en d'autres pays. Mais il est trop certain, nous venons de le rappeler par quelques exemples, qu'il lui manque une notion saine de la puissance de la science. Elle n'y croit pas, ou elle y croit trop tard.

En d'autres termes, l'esprit scientifique est moins répandu en France que dans d'autres contrées de l'Europe, moins répandu qu'en Amérique et qu'au Japon. L'industrie nationale a souffert profondément de ce défaut, et le manque d'esprit scientifique se fait sentir alleurs que dans l'industrie. Quel est la cause du mal?

Serions-nous moins bien doués que d'autres peuples pour la science? Il n'en est rien; l'histoire nous montre, de Cuvier à Claude-Bernard, de Lavoisier à Berthelot, que là, nous n'avons jamais quitté le premier rang. Le génie national n'a jamais cessé d'être inventif, original, doué d'initiative. Le mot et la chose sont français. Nous n'avons pas sujet d'accuser la nature

Il faut accuser notre instruction publique, qui ne connaît que la pédagogie de l'ancien régime et qui pèse bureaucratiquement sur l'enseignement supérieur.

Si l'on rencontre ici une ignorance, par moment impénétrable, ignorance bachelière et lettrée qui nous rappelle la Chine, la raison en est bien simple : notre pédagogie nous viènt de Chine. C'est là un fait historique. Notre pédagogie est celle de l'ancien régime, conservée par Napoléon. Elle sortit de l'ancien collège Lous-le-Grand, lequel fut fondé, on ne l'ignore pas, par des missionnaires revenus d'Extrême-Orient. On ne saurait d'ailleurs faire un reproche à ces missionnaires de s'être enthousiasmés pour le grand pays où ils pénétrèrent, de s'être plus qu'à moitié laissés convertir par les Chinois. Pendant que l'Europe était encore barbare et tourmentée, la Chine jouissait d'une civilisation paisible, prospère, encore brillante, munie d'organes qu'elle avait créés la première. Elle possédait déjà un système complet d'éducation, des classes régulières, une instruction publique administrée par des mandarıns. C'était néanmoins la culture naine : c'est-à-dire la science et la doctrine des adultes infligées à des écoliers, et, par contre, les adultes examinés, surve1llés et corrigés à la façon des jeunes élèves. La culture naine est la caricature de l'enseignement supérieur.

Ce système fonctionnait donc il $y$ a trois cents ans, et il nous vient si bien de là-bas, qu'aujourd'huı même, à l'heure où nous parlons, on ne trouve son analogue en aucun point du globe, sauf en France, en Espagne et en Chine.

Pour être tout à fait juste, il convient d'ajouter qu'il eût fallu une perspicacité singulière, ıl y a deux ou trois siècles, pour prévoir que la Chine allait devenir arriérée et routinière, qu'elle allait subir un arrêt complet de développement, causé par le fléau grotesque du mandarinisme.

Cet arrêt de développement a été complet et vraiment superbe parce que l'idéal de la cuistrerie avait été réalısé là complètement et purement. C'est un spectacle intéressant, parce qu'il est d'intérêt humain, et que la race n'y est pour rien.

Chez nous le même effet ne s'est produit que partiellement. Fort heureusement nous avons un enseignement supérieur. La Convention a créé des écoles savantes; puis les Universités sont nées, et la République a favorisé leur développement. C'est là qu'est le remède. L'enseignement supérieur a pour but de développer les facultés de l'adulte. Elle prend l'homme à vingt ans. Dans les pays où les conditions de la culture sont normales, on a soin d'envoyer à l'Université tous les jeunes gens auxquels on a la prétention de donner une instruction Jibérale, et cela ne fut-ce que par vanité et par respect humain, et en dehors de toute nécessité professionnelle. Cette nécessité d'une instruction supérieure n'est pas suffisamment comprise en France. On va bien à l'Université pour y faire son droit, sa médecine, etc., mais, pour former un homme instruit, on se contente de l'envoyer au collège. C'est se résigner à produire l'arrêt de développement à vingt ans; c'est faire de la culture naine

Le rôle de l'Université est surtout d'enseigner l'art de la recherche, ce qui veut dire la science, car la science est lart de la recherche, et pas autre chose. Et nous savons que la recherche est indispensable à l'Industrie. En même temps, l'Université est faite pour mettre les hommes qui n'ont pas d'ambitions scientifiques, mais qui veulent acquérir une culture générale digne de ce nom, en contact avec la science de première main, la seule qui soit attrayante etféconde, la seule qui soit libre de toute pédanterie.

Nos Universités ne sont pas entièrement en état de remplir cette double fonction. Elles sont encore soumises, tout comme l'enseignement' secondaire, à la loi napoléonienne. On sait que notre grand César, par raison politique sans doute, a écarté les projets de réforme soumis à la Convention, et qu'il a rétabli la pédagogie de l'ancien régime; on sait, en outre, qu'il a osé donner à son administration le pouvoir absolu de diriger 
les études, et en même temps la collation des grades Telle est l'origine du régime actuel; et aujourd'hui encore il n'existe pas, à côté du grand maître de l'Unıversité, un seul conseil, une seule commission ayant voix délibérative. Il serait temps que la République intervint, et qu'elle défit l'œuvre de Napoléon, avec le même soin qu'il mit à la farre. Il y a urgence à délivrer l'enseignement du pédantisme bureaucratique, et à libérer les universités du joug du pouvorr exécutif. Car celuici n'a pas cessé de peser sur les études supérieures en leur imposant sa pédagogie d'ancien régime.

Viendra-t-il jamais un grand ministre, ou une grande assemblée, pour retirer au pouvoir exécutıf la collation des grades et pour rendre ce droit à la seule autorité compétente?

Ici, nous sommes étonnamment en retard sur le reste du monde, l'Espagne exceptée. Où voit-on ailleurs une administratıon délivrant des diplômes scientıfiques? En Russie, certes, la bureaucratie jouit d'un pouvorr absolu, elle s'entend mal avec les universités, elle les licencie au besoin; mais elle n'empiète pas sur leurs attributions. A Saint-Pétersbourg, un ministre n'a pas le prestige nécessarre pour faire un docteur. I1 est vrai que les Russes sont un peuple jeune; comme ils n'ont pas à porter les deux mille ans de notre histoire, on ne peut s'attendre à rencontrer pari eux le gallo-romain enchinoisé.

e conclus en émettant le vœu, au nom de l'Industrie et du développement national, que l'enseignement de la science soit délivré des entraves anciennes, et je souhaite, en particulier, à l'Université de Lyon, l'autonomie et la prospérité qu'elle n'a pas cessé de mériter.

\section{Sur le Monvement des Liquides à grande vilesse dans les Condnites très larges \\ Note de M. Merczyng, à l'Académie des Sciences Séance du 14 janver 1907}

Le phénomène du mouvement d'un liquirle dans les tuyaux peut être envisagé de deux façons différentes. Si la conduıle présente un capillaire assez long, les lols du mouvement sont très simples, elles sont données par la théorie de M. Poiseulle (Mémoires des savants étrangers 1846).

Mais, si le tuyau devient large, on établit la lraison, entre la pente hydraulique par unilé de longueur du tuyau et la vitesse moyenne, par la vole expérimentale. Les travaux de M. Darcy (Mémoires des savants étrangers, 1857) ont fondé nos connaissances sur cetle question, pour les vitesses ne surpassant pas $3 \mathrm{~m}$. par seconde. Pour ces vitesses, Darcy a établı, après ses expériences, la formule binomiale.

D'un autre côté Darcy, et, d'une façon plus précise, M. Maurıce Lévy (1867) ont donné des expressions des pentes hydrauliques en fonction du diamètre du tuyau.

Mais, si les vitesses dépassent les valeurs employées dans le service des eaux ( 2 à $3 \mathrm{~m}$.), et si les conduites sont très larges $(0 \mathrm{~m} 40$ à $0 \mathrm{~m} 50)$, il n'existe pas de données expérimentales exactes pour les pentes hydrauliques, correspondant aux grandes vitesses. Les expériences de Darcy donnent les pentes pour un tuyau de $0 \mathrm{~m} 50$, seulement pour une vitesse de $1 \mathrm{~m}_{13}$. Si l'on vent donc se servir de la formule de Darcy, il faut de l'extrapolation, qui est très douteuse, vu le caractère très complexe du phénomène dans ce cás. Ayant à ma disposition des pompes centrifuges à vapeur de quelques centaines de chevaux, et deux condurles flottanles d'une longueur de $200 \mathrm{~m}$. chaque, dont l'une d'un diamètre intérıeur de $0 \mathrm{~m} 50$, et l'autre de $0 \mathrm{~m} 38$, j'entrepris quelques séries de mesures expérimentales des pentes hydrauliques dans ces tuyaux, pour les vitesses comprises entre 2 m8 et $4 \mathrm{~m} 5$ par seconde. Les conduites étaient en acier, sans dépôt, composées de tronçons de $7 \mathrm{~m} 5$, de longueur, sur des flotteurs; les raccordements étalent d'un même dıamètre, flexibles, en cuir. Les vitesses moyennes élaient mesurées par les débits dans un temps donné; les pentes par les manomètres, controlés par les piézomètres. Les conduites étaient alignées en ligne droite, et dans un même niveau horizontal.

Voici le résultat des mesures.

$$
\text { Moyenne Vitesse }
$$

Pente

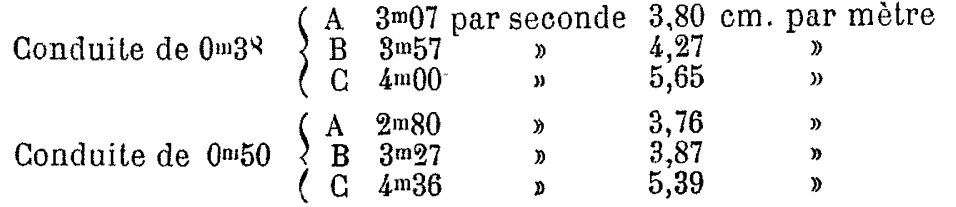

Les expériences donnent des résultats beaucoup plus élevés que les extrapolations de la formule de Darcy. Par exemple, pour la mesure A du tuyau de $0 \mathrm{~m} 50$, les lables de Darcy indiquent seulement une penle 0m0167, c'est-à-dire plus de deux fors plus petıle.

On peut donc conclure que, pour les conduites d'un diamètre surpassant $0 \mathrm{~m} 30$, et pour les vitesses moyennes plus grandes que $2^{\text {m5 }} 5$ à $3 \mathrm{~m}$., les pentes sont plus grandes que les valeurs indiquées par les extrapolations de la formule binomale de Darcy. ll est très probable que la lor qu lie les pentes à la rutesse n'esi, plus la formule binomiale, mais une expression exponenlielle, beaucoup plus compliquée. En tout cas, nos données expérimentales confirment la théorie de MM. Darcy el Maurice Lévy, que la pente augmente avec la diminution du diamètre du tuyau.

Si l'eau se meut à une vitesse de $3 \mathrm{~m}$. à $4 \mathrm{~m}$., les malières solides peuvent, par le phẻnomène hydrodynamique bien connu, rester en suspension dans le tuyau en quantité donnée, et l'on peut les transporter avec l'eau. Pour les vitesses indiquées, l'eau peut transporter jusqu'à 20 pour 100 du sable pur en suspension. Il était aussi très intéressant d'éludier les pentes hydrauliques pour ces mélangcs, ce que j'ai falt dans une autre série de mesures, dont les données suivent :

\begin{tabular}{|c|c|c|c|c|}
\hline & & $\begin{array}{l}\text { Vilesse } \\
\text { moyenne }\end{array}$ & $\begin{array}{l}\text { Quinnute } \\
\text { de siblele }\end{array}$ & $\begin{array}{l}\text { Pente en cm } \\
\text { par mètres }\end{array}$ \\
\hline Gonduile de 0 m38 & $\left\{\begin{array}{l}A \\
B \\
C\end{array}\right.$ & $\begin{array}{l}3 m+8 \\
3^{m+10} \\
3^{m}{ }^{m 8}\end{array}$ & $\begin{array}{l}12,8 \% \\
15,0 \\
11,5\end{array}$ & $\begin{array}{l}3,96 \\
4,12 \\
5,88\end{array}$ \\
\hline Conduite de $0 \mathrm{~m} 50$ & $\left\{\begin{array}{l}A \\
B \\
C \\
D \\
E\end{array}\right.$ & $\begin{array}{l}2 m 87 \\
3 m 20 \\
3 m+7 \\
3 m 60 \\
3 m 86\end{array}$ & $\begin{array}{l}12,6 \% \\
16,7 \\
13,5 \\
18,8 \\
16,7\end{array}$ & $\begin{array}{l}4,10 \\
4,12 \\
4,35 \\
4,60 \\
5,87\end{array}$ \\
\hline
\end{tabular}

Ces données établissen $l$ que $1^{0}$ la peute pour les mélanges esl, pour les mèmes vitesses, plus grande que pour l'eau pure, mais dans une mesure très étrolle; les mélanges de 12 à 18 pour 100 de sable donnent un accroissement de la pente seulement de 10 à 20 pour $100 ; 20$ la pente pour les mélanges plus riches en sable est plus grande que pour les pauvres. Par exemple, la pente pour la vitesse de $3 \mathrm{~m} 30$ est plus grande dans le grand tuyau que pour presque la même vilesse ( $3 \mathrm{~m} 18$ ) lans le pelit tuyau, mais la quanLité de sable est 16,7 pour 100 au lieu de 12,8 pour 100 .

Les données expérımentales que nous avons reprodulles peuvent servir comme hase pour le calcul du mouvement de l'eau ch des mélanges sableux.

\section{Déiermination de l'éehauffement d'un conducteur électrique}

Si un conducteur présente à la température $0_{1}$ une résistance $R_{1}$, et qu'il s'échauffe de 0 degré, sa résistance $R_{2}$ à la température $0_{1}+0$ sera donnée par la formule:

$$
R_{2}=R_{1}(\mathrm{I}+\%(0)
$$

$\alpha$ est un coefficient qui varie avec la température initiale $0_{1}$ et que M. A. E. Kennely a établi pour le cuivre, pour des températures initiales comprises entre $f^{\circ}$ el $50^{\circ}$ centigrades. Nous reproduisons ci-dessous le tableau donnant donnant ces valeurs de $\alpha$
$\begin{array}{lc}0 & * \\ 0 & 0.004200\end{array}$
$\begin{array}{ll}0 & 0.004200 \\ 1 & 0.004181\end{array}$
$0 \quad \alpha$
130.003983
11. 0.003967
20.001165
$\begin{array}{ll}3 & 0.004148\end{array}$
40.004131
$15 \cdot 0.003951$
$16 \quad 0.003936$
$17 \quad 0.003920$
$18 \quad 0.003905$
50.001114
$6 \quad 0.004097$
70.001080
$\begin{array}{ll}8 & 0.004063\end{array}$
$\begin{array}{ll}9 & 0.004047\end{array}$
$10 \quad 0.004031$
$11 \quad 0.004015$
$19 \quad 0.003890$
$20 \quad 0.003875$
$21 \quad 0.003860$
$22 \quad 0.003845$
$23 \quad 0.003830$
$24 \quad 0.003815$

$\begin{array}{rc}0, & \alpha \\ 26 & 0.003786 \\ 27 & 0003772 \\ 28 & 0.003758 \\ 29 & 0.003744 \\ 30 & 0.003730 \\ 31 & 0.003716 \\ 32 & 0.003702 \\ 33 & 0.003689 \\ 34 & 0.003675 \\ 35 & 0.003662 \\ 36 & 0.003648 \\ 37 & 0.003635 \\ 38 & 0.003622\end{array}$
$01 \%$
$39 \quad 0.003609$
10) 0.003596
410.003583
$\begin{array}{ll}12 & 0.003570\end{array}$
$430.00355 \%$
4.1 0.003545
$15 \quad 0.003532$
460.003520
$47 \quad 0.003508$
$48 \quad 0.003495$
$49 \quad 0.008483$
$50 \quad 0.003471$

250.003801

Cette forme spéciale présente un intérêt tout particulier pour la détermination de l'échauffemennt d'un conducteur en cuivre traversé par un courant électrique. 
Supposons, par exemple, que la résistance de ce conducteur soit de $0,23 \mathrm{ohm}$. à la température initiale $0_{1}$ de $25^{\circ}$, et qu'après le passage du courant elle devienne égale à $0,27 \mathrm{I}$ ohm. La formule (I) donne:

$$
0=\frac{\frac{R_{2}}{R_{1}}-1}{\alpha}
$$

En portant dans la formule (2) les valeurs indiquées on a :

$$
\frac{R_{2}}{R_{1}}=\mathrm{I}, \mathrm{I} 783 ; \quad \frac{R_{2}}{R_{1}}-\mathrm{I}=0,1783
$$

Et en divisant $0,17^{83}$ par la valeur de $\alpha$ correspondant à la température initiale de $25^{\circ}(x=0,00380 \mathrm{I})$, on en tire :

$$
0=\frac{0,1783}{0,003801}=46,9 \text { degrés }
$$

(Industrie éleclrique.)

\section{LE MOIS HYDRO-ÉLECTRIQUE}

\section{ACADÉMIE DES SCIENCES}

\section{GHIMIE ET ÉLEGTROGHIMIE}

Sur la distillation des alliages d'argent et de cuivre, d'argent et d'étain, d'argent et de plomb. - Note de MM. Henri Moissan et Tosio Watanabe. Séance du 7 janvier 1907.

Dans une série de recherches sur la distillation des corns simples, l'un de nous (") á fixé les conditions de l'ébullition d'un certain nombre de métaux, ainsi que les propriétés de ces éléments condensés sur une paroi troide. Ces expériences, répétées sur différents corps simples dans les mèmes conditions, ont fourni d'intéressantes comparaisons; il nous a paru utile, pour les vérıfier, d'étudier au four électrique la distrllation d'un certain nombre d'alliages.

Une première étude a été réalisée sur ce sujet par MM. Morssan et O'Farrelley $\left({ }^{*}\right)$, qui avaient envisagé la distıllation des alliages que le cuivre fournt avec le zınc, le cadmium, le plomb et l'étain. Il s'agissait, dans ce cas, de métaux très tacllement volatils. Nous avons entrepris l'etude de la distillation des allages d'argent et de cuivre, d'argent et d'étain, d'argent et de plomb. En prenant ainsi différents allages ayant un metal commun, et en multipliant les expériences, on peut obtenir un ensemble de résultats comparables.

l.es expériences que nous donnons dans cette note ont été realisées dans le four élestrique à tube décrit anterteurement. Nous rappellerons que les tubes et les nacelles de graphite que nous employons sont aussi purs que possible, et ne doivent jamais contenir plus de I pour 100 de cendres. Les métaus prénarés specialement pour ces recherches ont éte fondus au préalable dans des proportions déterminees, vérifiées ensulte par des analyses. Une nouvelle analıse portant sur plusieurs fragments du lingot recueillı après l'exnérience permet de se rendre compte de la quantité de chaque metal qui reste dans la nacelle et, par différence, des quantités distillées.

Allage d'argent et de cuivre - Nous sommes partis d'un alliage de cuivre et d'argent qui renfermat, à peu pres, des port ons égales de ces deux métaux, et nous avons fait varier le temps de chauffe de I minute à 15 mınutes, ansi qu'il $r$ ssort du premier tableau ci-joint.

Le début de l'expérience datait du moment où l'alliage était liquade. Le courant étart maintenu aussi constant que possible dans les environs de 500 ampères sous 110 volts. La volatilisation commence après 35 secondes et, après 3 minutes de distillation, une certaine quantité de graphite est déjá entrée en solution dans l'allıage.

Pendant les trois premières minutes, les quantités d'argent restant dans la nacelle varient peu, et la quıntute de vapeur produite par la distillation peut être regardée à peu pres comme constante jusqu'à la sixteme minute. Puis elle croit brusquement pendant que la quantité d'argent dıminue dans l'alliage.

(") H. Moissan, Sur la distillation des corps simples. Ann. de ch. et de ph., 8. serte, t. VIII, ir,06, p it 5 .

("*) G. Moissan et O'Farrelley, Comptes rendus, t. CXXXvill 1904, p. 1659 .
Il est possible qu'à partir de la quatrième minute, il se forme un allıage défini, mais il ne serait stable qu'à l'état lıquide, car la métallographie et l'étude physique des alliages de curve et d'argent n'ont indiqué l'exiitence d'ducun composé défini de ces deux métaux.

Il ressort de cet ensemble d'expériences que, plus la distillation est longue, plus l'alliage restant s'enrichit en cuivre Nos expériences démontrent donc que le point d'ébullıtion du curvre est plus élevé que celui de l'argent. Nous sommes partis d'un alliage à 49,55 d'ar-

\begin{tabular}{|c|c|c|c|c|c|c|c|c|}
\hline \multirow{3}{*}{$\begin{array}{c}\text { Durée } \\
\text { de la } \\
\text { clraufe }\end{array}$} & \multirow{3}{*}{$\begin{array}{l}\text { Pords } \\
\text { de l'alliage } \\
\text { inital }\end{array}$} & \multirow{3}{*}{$\begin{array}{l}\text { Alliage } \\
\text { restant }\end{array}$} & \multirow{3}{*}{ Différences } & \multirow{3}{*}{$\begin{array}{c}\text { Perle } \\
\text { de } \\
\text { Poids }\end{array}$} & \multicolumn{4}{|c|}{ Composition de l'alliage } \\
\hline & & & & & \multicolumn{2}{|c|}{ Intial } & \multicolumn{2}{|c|}{ Final } \\
\hline & & & & & Ag. & $\mathrm{Cu}$. & $\mathrm{Ag}$. & $\mathrm{Cu}$. \\
\hline min. & $\mathrm{gr}$ & $\mathrm{gr}$ & $\mathrm{gr}$ & $\%$ & $\%$ & $\%$ & $\%$ & $\%$ \\
\hline 1 & 39,1434 & 38,2113 & 0,9321 & 3,00 & 48,85 & 51,04 & 49,86 & 50,64 \\
\hline 2 & $39, ; 698$ & 38,9113 & 0,8585 & 2,16 & $"$ & $"$ & 49,29 & 50,89 \\
\hline 3 & 40,5854 & 36,12 & $\begin{array}{l}4,4636 \\
\end{array}$ & 11,00 & & $"$ & 45,11 & 54,63 \\
\hline ü & 40,10604 & $\mathbf{5 5 , 6 2 1 5}$ & $\begin{array}{l}4,4380 \\
4,4309\end{array}$ & 11,08 & 49,12 & 51,28 & 47,42 & 52,02 \\
\hline 5 & 40,5914 & 220588 & 18,5356 & 36,28 & 1 & $\nabla$ & 20,91 & 78,64 \\
\hline & $10,31 !$ & 20,8413 & 19,4781 & 48,31 & in & " & 17,98 & 81,34 \\
\hline 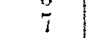 & 36,1314 & 21,65 & 15,4770 & 42,81 & " & " & 18,54 & 8127 \\
\hline 9 & $38,9:$ & $2 h, 4 i$ & 12,4849 & 30,60 & 49,55 & 49,65 & 1833 & 80,70 \\
\hline 13 & 39,5539 & 13,6110 & $25,9 \mathfrak{i} 39$ & 65,59 & 49,55 & 49,65 & 4,77 & 94,43 \\
\hline 15 & 39),34114 & $6,7740^{\circ}$ & 32,5664 & 82,77 & 0,00 & 1 & 3,62 & 95,22 \\
\hline
\end{tabular}
gent et à 49,65 de cuivre et, après 15 minutes de chauffe, nous avons obtenu un alliage à 3,62 d'argent et à 95,22 de cuivre.

Alliage d'argent et d'étain. - Dans une note précédente, MM. Moissan et O'Farrelly ont établi que le point d'ébullition de l'étain est plus élevé que celut du cuivre. Il était intéressant de chercher s'il existe une différence notable entre le point d'ébullition de l'étain et celui de l'argent. Nous sommes partis d'un alliage initial qui contenait 36,98 d'argent et 69,04 d'étain. (iet alliage a été chauffé dans les memes conditions que celut d'argent et de cuivre, et la volatilisation a commencé io secondes après la fusion. L.es durées de chauffe ont été de 2 , de 6 et de 10 minutes. Dans l'expérience la plus longue, celle de ro minutes. la composition du résidu non distıllé était de : argent, 2,93 ; étain, 93,65 . Dans ces expériences, l'ar-

\begin{tabular}{|c|c|c|c|c|c|c|c|}
\hline \multirow{3}{*}{$\begin{array}{l}\text { Durée } \\
\text { de la } \\
\text { chauffee }\end{array}$} & \multirow{2}{*}{\multicolumn{2}{|c|}{ Alliage }} & \multirow{3}{*}{ 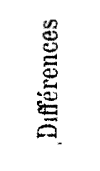 } & \multicolumn{4}{|c|}{ Composition } \\
\hline & & & & \multicolumn{2}{|c|}{ Allage inttial } & \multicolumn{2}{|c|}{ Alliage final } \\
\hline & Inittal & Restant & & $\Lambda \mathrm{g}$. & Sn. & Ag. & $\mathrm{Sn}$ \\
\hline min. & gr. & gr. & gr. & $\%$ & $\%$ & $\%$ & $\%$ \\
\hline 2 & 40.7328 & 396966 & 1,0362 & 36,98 & 64,04 & 33,72 & 65,53 \\
\hline $\begin{array}{c}6 \\
10\end{array}$ & $\begin{array}{l}40,7481 \\
39,0705\end{array}$ & $\begin{array}{r}17.8956 \\
9,4836\end{array}$ & $\begin{array}{l}22.8525 \\
29,5869\end{array}$ & ") & 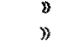 & $\begin{array}{r}10,14 \\
2,83\end{array}$ & $\begin{array}{l}88,81 \\
93,65\end{array}$ \\
\hline
\end{tabular}
gent, de même que le culvre, possède une température d'ébullition moins élevée que celle de l'étain.

Alliage d'argent et de plomb. La rapidité de la distillation du plomb dans les conditions. où nous étions placés était telle que nous avous dû diminuer considérablement le temps de chaufte. Les trois expériences que nous mentionnons ont été faites avec un alliage initial renfermant 46,24 d'argent et 52,95 de plomb; elles n'ont duré que 1,2 et 2 minutes I/ 2 Dans la dernière expérience, la quantité d'argent restant dans le culot était de 93,81 pour too; par conséquent, non seulement le plomb distille beaucoup plus, vite que l'ar-

\begin{tabular}{|c|c|c|c|c|c|c|}
\hline \multirow{3}{*}{$\begin{array}{l}\text { Durée } \\
\text { de la } \\
\text { chauffe }\end{array}$} & \multirow{2}{*}{\multicolumn{2}{|c|}{ Alliage }} & \multirow{3}{*}{ 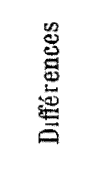 } & \multicolumn{3}{|c|}{ Composition } \\
\hline & & & & \multicolumn{2}{|c|}{ Alliage initial } & \multirow{2}{*}{$\begin{array}{c}\text { Alliage } \\
\text { final } \\
\text { Ag. }\end{array}$} \\
\hline & Intital & Final & & Ag. & $\mathrm{Ag}$ & \\
\hline $\min$ & $\mathrm{gr}$. & g̨r. & gr. & $\%$ & $\%$ & $\%$ \\
\hline 1 & 40,9330 & 29,6775 & 12,2955 & 16,24 & 52,95 & 60,75 \\
\hline 2 & 38,7832 & 15,1460 & 25.6392 & 17 & $"$ & 83,06 \\
\hline 2,30 & 42,8045 & 4,3244 & 38,4801 & " & $》$ & 96,81 \\
\hline
\end{tabular}
gent, mais encore l'alliage argent et plomb distille plus rapidement que l'alliage argent et cuivre.

Conclusions - Nous devons faire remarquer que ces expériences, faites dans des temps égaux, toutes les autres conditions étant constantes (ampérage, voltage, diamètre des électrodes, 'capacité du four) ne sont pas cependant entièrement comparables. Il va de soi que la quantité de chaleur fournie par l'arc dans un appareil froid, ou dans 